\title{
SOMETIMES ONLY SQUARE MATRICES CAN BE DIAGONALIZED
}

\author{
LAWRENCE S. LEVY
}

ABSTRACT. It is proved that every SQUARE matrix over a serial ring is equivalent to some diagonal matrix, even though there are rectangular matrices over these rings which cannot be diagonalized.

Two matrices $A$ and $B$ over a ring $R$ are equivalent if $B=P A Q$ for invertible matrices $P$ and $Q$ over $R$.

Following R. B. Warfield, we will call a module serial if its submodules are totally ordered by inclusion; and we will call a ring $R$ serial if $R$ is a direct sum of serial left modules, and also a direct sum of serial right modules. When $R$ is artinian, these become the generalized uniserial rings of Nakayama [E-G].

Note that, in serial rings, finitely generated ideals do not have to be principal; for example, let $R$ be any full ring of lower triangular matrices over a field. Moreover, it is well known (and easily proved) that if $a R+b R$ is a nonprincipal right ideal in any ring, the $1 \times 2$ matrix $[a b]$ is not equivalent to a diagonal matrix. Thus serial rings do not have the property that every rectangular matrix can be diagonalized.

1. Background. Serial rings are clearly semiperfect $(R / \mathrm{rad} R$ is semisimple artinian and idempotents can be lifted modulo $\operatorname{rad} R$ ).

(1.1) Every indecomposable projective module over a semiperfect ring $R$ is $\cong e R$ for some primitive idempotent $e$ of $R$. (See [M].)

A double-headed arrow $\rightarrow$ will denote an "onto" homomorphism.

By a projective cover of a module $X$ we mean a minimal epimorphism $\phi$ : $P \rightarrow X$ with $P$ projective. ("Minimality" means that no submodule other than $P$ itself is mapped onto $X$; equivalently, $\operatorname{ker} f$ is superfluous in $P_{\text {.) }}$ )

(1.2) (Uniqueness of the projective cover). Let $f: P \rightarrow X$ be a module epimorphism with $P$ projective, and suppose that $X$ has a projective cover $\phi: P_{1} \mapsto X$. Then $P=P^{\prime} \oplus P^{\prime \prime}$ where $f\left(P^{\prime \prime}\right)=0$ and $f: P^{\prime} \rightarrow X$ is a projective cover. Moreover, there is an isomorphism $\theta: P^{\prime} \rightarrow P_{1}$ such that $\left(f \mid P^{\prime}\right)=$ $\phi \theta$. (See [B, 2.3].)

(1.3) Over a semiperfect ring, every finitely generated module has a projective cover [B, 2.1].

Received by the editors July 17, 1974 .

AMS (MOS) subject classifications (1970). Primary 16A64, 16A46, 15A21, 15A33; Secondary 16A50.

Key words and phrases. Matrix, matrix equivalence, diagonal matrices, generalized uniserial ring, serial ring, projective module. 
(1.4) If $\phi_{i}: P_{i} \rightarrow X_{i}$ is a projective cover of $X_{i}$ over any ring ( $i=1$, $\ldots, n)$, then

$$
\phi=\bigoplus_{i=1}^{n} \phi_{i}: \bigoplus_{i=1}^{n} P_{i} \rightarrow \bigoplus_{i=1}^{n} X_{i}
$$

is a projective cover of $\bigoplus X_{i}$.

Proof. Since $X_{i}$ is finitely generated, minimality of $\phi_{i}$ shows that $P_{i}$ is finitely generated, too. Hence we easily establish: $\operatorname{ker} \phi_{i}$ is superfluous in $P_{i} \Leftrightarrow$

$\operatorname{ker} \phi_{i} \subseteq \operatorname{rad} P_{i}=\bigcap\left(\right.$ all maximal submodules of $\left.P_{i}\right)$

(This is more or less [B0, p. 64, Corollary 3].) Then use the fact that $\operatorname{rad}\left(\bigoplus P_{i}\right)=\bigoplus \operatorname{rad} P_{i}[$ BO, p. 64, Corollary 2].

2. Theorem. Let $\alpha: P \rightarrow Q$ be a homomorphism of finitely generated, projective right modules $P$ and $Q$ over a serial ring $R$. Then there exist decompositions $P=P_{1} \oplus \cdots \oplus P_{s}$ and $Q=Q_{1} \oplus \cdots \oplus Q_{t}$ with each $P_{i}$ and $Q_{i}$ indecomposable, such that

$$
\alpha\left(P_{i}\right) \subseteq Q_{i} \quad \text { for } i \leq w \text { and } \alpha\left(P_{i}\right)=0 \quad \text { for } i>w
$$

where $w=\min \{s, t\}$.

Proof. This theorem is a slight extension of Warfield's theorem [W, 3.3], which states that if $X$ is a finitely generated submodule of a finitely generated projective module $Q$ over a serial ring $R$, then there is a decomposition

$$
Q=Q_{1} \oplus \cdots \oplus Q_{t} \quad\left(\text { each } Q_{i}\right. \text { indecomposable) }
$$

such that

$$
X=X_{1} \oplus \cdots \oplus X_{t} \quad\left(\text { each } X_{i}=X \cap Q_{i}\right)
$$

Choose the numbering so that $X_{i}$ is nonzero for $i \leq w$ and $X_{i}=0$ for $i>w$.

Now let $P$ be the module in our theorem, and $X=a(P)$. Each $X_{i}$ in (3) is finitely generated, because it is a homomorphic image of the finitely generated module $X$, and therefore has a projective cover $\phi_{i}: P_{i} \rightarrow X_{i}$. Moreover, $X_{i}$ is serial $\left(X_{i} \subseteq\right.$ the serial module $Q_{i}$ ) and therefore $P_{i}$ is indecomposable. (If $P_{i}=A \oplus B$, then $X_{i}=\phi_{i}(A)+\phi_{i}(B)$. Since $X_{i}$ is serial we can suppose $\phi_{i}(A) \supseteq \phi_{i}(B)$. Then $X_{i}=\phi_{i}(A)$ and by "minimality" of the projective cover, $A=P_{i}$.)

By (1.4),

$$
\bigoplus_{i=1}^{u} \phi_{i}: \bigoplus_{i=1}^{u} P_{i} \rightarrow \bigoplus_{i=1}^{w} X_{i}
$$


is a projective cover of $X$; and by uniqueness of the projective cover (1.2), we can suppose that $\bigoplus_{i} P_{i}$ is a direct summand of $P$ (each $\phi_{i}$ then becomes the restriction of $a$ to $P_{i}$ ) with the complimentary summand being mapped by $a$ to 0 .

Thus the theorem is proved except that the $w$ obtained above might be $<\min \{s, t\}$. But, when $i>$ the $w$ we have obtained, $a\left(P_{i}\right)=\{0\} \subseteq Q_{i}$ provided, of course, that $i \leq \min \{s, t\}$. Thus we can change to $w=\min \{s, t\}$. Q.E.D.

3. Main Theorem. Every square matrix over a serial ring is equivalent to some diagonal matrix.

Proof. Since $R$ is serial, it has a decomposition

$$
R=e_{1} R \oplus \cdots \oplus e_{d} R
$$

with $\left\{e_{i}\right\}$ an orthogonal set of primitive idempotents.

Let $\alpha: R^{(n)} \rightarrow R^{(n)}$ be left multiplication by the given $n \times n$ matrix $A$, elements of $R^{(n)}$ being written as columns. It will suffice to write $R^{(n)}=$ $R_{1}^{\prime} \oplus \cdots \oplus R_{n}^{\prime}=R_{1} \oplus \cdots \oplus R_{n}$, with each $R_{i}^{\prime}$ and $R_{i} \cong R_{R}$, and each $a\left(R_{i}^{\prime}\right)$ $\subseteq R_{i}$

The first step is to write $R^{(n)}$ as a direct sum of indecomposable modules $P_{k}$ and $Q_{k}$ in such a way that $a$ takes the form

$$
\alpha: R^{(n)}=\bigoplus_{k=1}^{n d} P_{k} \rightarrow R^{(n)}=\bigoplus_{k=1}^{n d} Q_{k} \quad \text { with } \alpha\left(P_{k}\right) \subseteq Q_{k}
$$

where $d$ is the integer defined in (1). To do this, merely apply Theorem 2, with $P=Q=R^{(n)}$. To see that $k$ runs from 1 through $n d$, as claimed in (2), note that the ring of endomorphisms of $e_{i} R$ is $e_{i} R e_{i}=$ local since $R$ is semiperfect [L, p. 76, Proposition 2], and therefore the Azumaya-Krull-Schmidt theorem applies to decompositions of $R^{(n)}$ (see [L, p. 78, Corollary]).

We will have our submodules $R_{i}^{\prime}$ and $R_{i}$ if we can express the set $\{1,2$, $\ldots, n d\}$ as the union of $n$ disjoint subsets $S(1), \ldots, S(n)$ such that, for each $b$,

$$
\bigoplus_{k \in S(b)} P_{k} \cong R \text { and } \bigoplus_{k \in S(b)} Q_{k} \cong R
$$

To do this, recall that

$$
\bigoplus_{k=1}^{n d} P_{k}=R^{(n)}=\left(\bigoplus_{i=1}^{d} e_{i} R\right)^{(n)}
$$

By the Krull-Schmidt theorem, there are at least $n$ indices $k$ such that $P_{k}$ $\cong e_{1} R$. (There will be more than $n$ such indices $k$ if, say, $e_{1} R \cong e_{2} R$.)

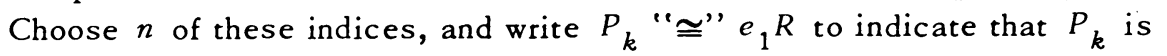
one of the "chosen" $P$ 's which are $\cong e_{1} R$. Then choose $n$ different $P_{k}$ 's 
"@" $e_{2} R$, and so on, until we partition $\left\{P_{k}\right\}$ into $d$ disjoint $n$-element subsets, each associated with a distinct $e_{i} R$ (and thus we exhaust the $e_{i} R$, too).

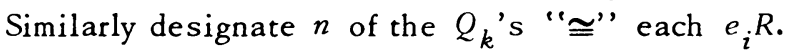

We can keep track of all this by introducing a set of ordered triples $(k, i \rightarrow j)$, one for each $k \in\{1,2, \ldots, n d\}$, to indicate that $P_{k}$ " $\cong$ "' $e_{i} R$ and

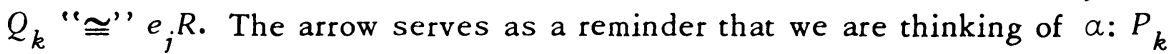
$\rightarrow Q_{k}$ as a map: $e_{i} R \rightarrow e_{j} R$.

Next, let $M$ be the $d \times d$ matrix of nonnegative integers whose $(i, j)$ entry is

$$
m_{i j}=\text { the number of triples of the form }(k, i \rightarrow j) \quad(k \text { varying })
$$

This matrix $M$ has two properties which interest us. For each $i$, the sum of the entries $\Sigma_{j=1}^{d} m_{i j}$ in row $i$ equals the number of $P_{k}$ which are " $\cong " ~ e_{i} R$; that is, $n$. Similarly the sum of the entries in each column equals $n$.

Now, a theorem of Birkhoff states that if a square matrix $M$ of nonnegative real numbers has all of its row sums and column sums equal to the same number, then $M$ is a sum $u_{1} M_{1}+u_{2} M_{2}+\cdots$ of nonnegative multiples of permutation matrices $M_{b}$. (A $d \times d$ permutation matrix is a square matrix which can be obtained by permuting the rows of the $d \times d$ identity matrix.) In fact, one can see by looking through the proof of Birkhoff's theorem given in $[\mathbf{H}$, p. 52], if the entries of $M$ are all nonnegative integers, we can choose the multipliers $u_{b}$ to be nonnegative integers, too. Writing this multiplication as repeated addition, we see that our matrix $M$ defined in (4) is a sum of permutation matrices. Checking the row sums then shows that $M$ is the sum of exactly $n$ permutation matrices

$$
M=M_{1}+\cdots+M_{n}\left(M_{b}=\text { permutation matrix }\right) .
$$

Now we build the sets $S(b)$. For each ordered pair $(i, j)$ such that $m_{i j} \neq 0$, the number of $k$ 's which occur in a triple $(k, i \rightarrow j)$ equals $m_{i j}$, and this also equals the number of matrices $M_{b}$ in $(5)$ whose $(i, j)$ entry equals 1 . Therefore we can choose a $1-1$ correspondence between the numbers $k$ in the set $\{1,2, \ldots, n d\}$ and the nonzero entries of the matrices $\left\{M_{b}\right\}$, such that $k \leftrightarrow$ $(i, j)_{b}=$ the $(i, j)$ entry of $M_{b}$ implies $P_{k} \cong e_{i} R$ and $Q_{k} \cong e_{j} R$. Then let $S(b)$ be the set of all $k$ 's which correspond to an entry of $M_{b}$, that is, $k \leftrightarrow$ $(,)_{b}$. Since a permutation matrix contains exactly one nonzero entry in éach row and one in each column, (3) holds, and the proof is complete.

4. Remark. We have not dealt with uniqueness of the diagonal matrices obtained here because, as observed in [L-R, 4.3], if $R$ is any semiperfect ring, $\operatorname{diag}\left(c_{1}, \ldots, c_{n}\right)$ is equivalent to $\operatorname{diag}\left(d_{1}, \ldots, d_{n}\right) \Leftrightarrow \bigoplus R / c_{i} R \cong \bigoplus R / d_{i} R$ (isomor phism of $R$-modules). 


\section{REFERENCES}

[B] H. Bass, Finitistic dimension and a homological generalization of semi-primary rings, Trans. Amer. Math. Soc. 95 (1960), 466-488. MR 28 \#1212.

[BO] N. Bourbaki, Éléments de mathématique. XXIII. Part 1. Les structures fondamentales de l'analyse. Livre II: Algèbre. Chap. 8: Modules et anneaux semisimples, Actualités Sci. Indust., no. 1261, Hermann, Paris, 1958. MR 20 \#576.

[E-G] D. Eisenbud and P. Griffith, Serial rings, J. Algebra 17 (1971), 389-400. MR $43 \# 2021$.

[H] Marshall Hall, Jr., Combinatorial theory, Blaisdell, Waltham, Mass., 1967. MR $37 \# 80$.

[L] J. Lambek, Lectures on rings and modules, Blaisdell, Waltham, Mass., 1966. MR $34 \# 5857$.

[L-R] L. S. Levy and J. C. Robson, Matrices and pairs of modules, J. Algebra 29 (1974), 427-454.

[M] B. J. Muller, On semi-perfect rings, Illinois J. Math. 14 (1970), 464-467. MR $41 \# 6909$.

[W] R. B. Warfield, Serial rings, semihereditary rings, and finitely presented modules, J. Algebra (to appear).

DE PARTMENT OF MATHEMATICS, UNIVERSITY OF WISCONSIN, MADISON, WISCONSIN 53706 